\title{
DESCENTRALIZACIÓN SUBNACIONAL, AUTONOMÍA Y DISPARIDADES FISCALES EN BRASIL
}

\author{
DESCENTRALIZAÇÃO SUBNACIONAL, AUTONOMIA E DISPARIDADE FISCAL NO BRASIL \\ SUBNATIONAL DECENTRALIZATION, AUTONOMY AND FISCAL DISPARITIES IN BRAZIL
}

\section{RESUMEN}

Este trabajo es un estudio aplicado de finanzas públicas subnacionales enfocado en los aspectos de descentralización, autonomía e incidencia fiscal territorial en Brasil. Se presentan mediciones y estimaciones de descentralización, autonomía e igualdad territorial de oportunidades (de gastos, ingresos propios y transferencias) en el sector subnacional y se evalúa el impacto regional de políticas fiscales alternativas. Los resultados muestran que los Estados y Municipalidades son un conjunto muy heterogéneo, que existen fuertes desequilibrios fiscales horizontales, que las transferencias tienen un componente distributivo que los atenúan, pero no los compensan totalmente, y que puede esperarse un rol creciente de los gobiernos municipales.

PALABRAS CLAVE: Federalismo, descentralización, autonomía, desigualdad fiscal, incidencia territorial.

\section{Alberto Porto ${ }^{1}$}

porto.alb@gmail.com

ORCID: 0000-0002-0333-2495

Walter Rubén Rosales ${ }^{1}$

walter75@yahoo.com

ORCID: 0000-0003-2382-3352

1 Universidad Nacional de La Plata, Facultad de Ciencias Económicas, La Plata, Buenos Aires, Argentina

Sometido 28.12.2018. Aprobado 10.06.2019.

Evaluado por el sistema double blind review.

DOI: http://dx.doi.org/10.12660/cgpc.v24n78.77994 


\section{RESUMO}

Este artigo é um estudo aplicado às financas públicas subnacionais, focado nos aspectos de descentralização, autonomia e incidência fiscal territorial no Brasil, sendo apresentadas medidas e estimativas dé descentralização, autonomia e igualdade territorial de oportunidades (de despesas, receitas próprias e transferências). O setor subnacional e o impacto regional de políticas fiscais alternativas são avaliados. Os resultados mostram que os estados e municípios são um grupo muito heterogêneo, que há fortes desequilíbrios fiscais horizontais, que as transferências têm um componente distributivo que as atenua, mas não as compensa totalmente, e que se espera um papel crescente dos governos municipais.

PALAVRAS-CHAVE: Federalismo, descentralização, autonomia, desigualdade fiscal, incidência territorial.

\section{ABSTRACT}

This paper is an applied study of subnational public finances focused on decentralization, autonomy and territorial fiscal incidence in Brazil. Measurements and estimates of decentralization, autonomy and territorial equality of opportunities (of expenditures, own revenues and transfers) in the subnational sector are presented and the regional impact of alternative fiscal policies is evaluated. The results show that States and Municipalities are a very heterogeneous group, there are strong horizontal fiscal imbalances, transfers have a distributive component that attenuates them but does not compensate them totally, and that a growing role of municipal governments can be expected.

KEYWORDS: Federalism, decentralization, autonomy, fiscal inequality, territorial incidence.

\section{INTRODUCCIÓN}

Este trabajo es un estudio aplicado de finanzas públicas subnacionales enfocado en los aspectos de descentralización, autonomía e incidencia fiscal territorial en Brasil. Brasil es un país con alto grado de descentralización dentro del grupo de países en desarrollo y el más descentralizado en América Latina -algo más que en México y la Argentina. La descentralización en Brasil tiene características que la diferencian de esos dos países y lo convierten en un caso de estudio de interés: a) la autonomía fiscal subnacional (recursos propios/gastos) es $61 \%$ siendo en Argentina del $15 \%$ y en México $12 \%$; b) la importancia relativa del gasto de los gobiernos municipales es del $23 \%$ y del $8 \%$ en los otros dos países; y c) las municipalidades forman parte de la Federación por disposición constitucional. Una característica común es la existencia de grandes disparidades regionales y la existencia de complejos sistemas de transferencias diseñados para enfrentarlos (para detalles ver Afonso y Serra,
2007, Porto, Pineda Mannheim, \& Eguino, 2018 y las demás referencias a Brasil).

El centro de la atención se ubica en la descentralización dentro del sector subnacional (participación de las municipalidades en el total subnacional) dado el proceso de "glocalización", que significa un rol creciente para las instituciones globales y los gobiernos locales y un rol menor para los gobiernos nacionales y estatales.

Se presentan mediciones y estimaciones de descentralización, autonomía e igualdad territorial de oportunidades en el sector subnacional (de gastos, ingresos propios y transferencias) y se evalúa el impacto regional de políticas fiscales alternativas. Se presenta también un marco conceptual, las cuantificaciones y estimaciones y finalmente una conclusión.

Los resultados muestran que los departamentos y municipalidades son un conjunto muy heterogéneo, que existen fuertes dese- 
quilibrios fiscales horizontales, que las transferencias tienen un componente distributivo que los atenúan pero no los compensan totalmente, y que puede esperarse un rol creciente de los gobiernos municipales. Se simulan alternativas de políticas diseñadas para disminuir los desequilibrios horizontales y se analizan los conflictos entre objetivos.

\section{MARCO CONCEPTUAL}

\section{Descentralización Fiscal}

Este trabajo pone la atención en la medición y análisis de la descentralización dentro del sector subnacional, o sea, la participación de las variables fiscales municipales (gastos, ingresos propios y transferencias) en el total de estados más municipalidades. Este tema es importante ya que el nuevo contexto internacional, caracterizado por la globalización y las revoluciones informática y tecnológica, afecta la distribución de los gastos y los recursos públicos entre los niveles de gobierno. Los efectos previstos se resumen en la expresión de Bell (1987): "the nation-state is becoming too small for the big problems of life, and too big for the small problems of life." Courchene (1993) ha denominado a ese cambio "glocalization", que significa un rol creciente para los gobiernos (instituciones) globales y los gobiernos locales, y un menor rol para los gobiernos nacionales y estatales.

\section{Autonomía fiscal}

Una de las cuestiones centrales que estudia la teoría del federalismo fiscal es la asignación de potestades tributarias y de responsabilidades de gasto entre niveles de gobierno. De esas asignaciones resultan, en general, diferentes composiciones de ingresos y gastos. Aparece así el desequilibrio vertical en el sector público multinivel: aun cuando a nivel consolidado los ingresos y los gastos gubernamentales sean iguales, los gobiernos subnacionales (GSN) usualmente registran una brecha entre gastos e ingresos propios (déficits) en tanto que el gobierno nacional suele registrar el comportamiento contrario (superávits).

La autonomía fiscal se refiere al poder de decisión de los GSN en cuanto a gastos, ingresos y financiamiento. En este estudio, la autonomía se define como el cociente RP/ GT donde RP son los recursos propios y GT el gasto total subnacional. Este indicador, conocido también como "correspondencia fiscal media", mide el grado en que un GSN puede financiar sus gastos con recursos propios. Su valor varía entre cero (dependencia total para financiar sus gastos) y uno (autofinanciamiento). La máxima autonomía corresponde a la capacidad de los GSN de elegir las bases y alícuotas de sus impuestos. En una situación intermedia, el GSN tiene autonomía para fijar las alícuotas sobre bases definidas por el gobierno central. La mínima autonomía corresponde a la situación en que las decisiones sobre las bases y alícuotas corresponden al gobierno central (GC).

Sobre la importancia de la autonomía de los GSN hay distintas visiones en las teorías del federalismo fiscal. En las teorías de primera generación se asume que los gobiernos son benevolentes y que buscan la eficiencia y la equidad regionales. Las transferencias intergubernamentales tienen un rol importante ya que cubren el desequilibrio vertical que resulta de la asignación eficiente de recur- 
sos y gastos entre niveles de gobierno. Las transferencias son también un instrumento para corregir los efectos de los desequilibrios económicos regionales y reducir los residuos fiscales. El residuo fiscal será diferente sin transferencias ya que en la región "pobre", para proveer la misma cantidad de bienes que en la región "rica" será necesaria una mayor presión tributaria, o con igual presión tributaria, la cantidad de bienes provista será menor. La autonomía local amplia puede originar trabas al comercio interjurisdiccional y diferentes residuos fiscales para los habitantes de las distintas regiones. Las diferencias de residuos fiscales pueden alentar migraciones de personas y de capital (Buchanan, 1950).

Una visión diferente presenta los desarrollos de las teorías del federalismo fiscal de segunda generación. En este caso pueden visualizarse dos avenidas de análisis. La predominante es la que enfatiza "un lado malo" de la descentralización fiscal: las transferencias generan una restricción presupuestaria blanda que lleva a comportamientos perversos de los GSN que culminan con la crisis de todo el sector fiscal y de la economía del país. En la segunda avenida aparece el "otro lado malo" de las transferencias: el GC concentra los recursos fiscales y los utiliza como medio de control político de los GSN. En esta visión más amplia los comportamientos destructivos pueden surgir del GC y/o de los GSN (Oates, 2005, Rezende, 2016). Weingast (1995) agrega la relación entre el crecimiento económico y el modelo de federalismo que posibilita el buen funcionamiento de los mercados ("market-preserving federalism"). El federalismo preservador del mercado genera incentivos para que los GSN inviertan sus recursos en forma eficiente para mejorar su posición competitiva (Qian \& Weingast, 1997; Weingast, 1995, 2009). Una combinación de estos argumentos reforzó la importancia de la autonomía fiscal de los GSN y provocó un cambio en las políticas sugeridas para la asignación de gastos y recursos. La secuencia para organizar la estructura vertical del sector público cambia: primero se asignan los gastos y luego los impuestos con la finalidad de lograr una restricción presupuestaria fuerte. El punto clave en esta visión es que para que los GSN provean la cantidad eficiente de los bienes públicos subnacionales es necesario que recauden sus propios impuestos. Cuáles son los impuestos son los más adecuados es un tema de gran complejidad (Bird, 2010).

\section{Aspectos distributivos regionales}

En general, los GSN tienen jurisdicción no sólo sobre grupos de personas con preferencias distintas sino también sobre territorios con distintas bases imponibles y distintos costos de provisión de los servicios públicos. Existen regiones ricas y pobres, con costos de provisión relativamente altos y bajos. Estos dos hechos pueden originar migraciones ineficientes (inducidas fiscalmente) si es que no se corrigen (vía transferencias intergubernamentales) de modo de igualar los residuos fiscales (Buchanan, 1950). Además de este argumento de eficiencia económica para la igualación de residuos fiscales, existen las cláusulas constitucionales y/o legales que establecen el principio de igualdad de oportunidades o igualdad de provisión de ciertos bienes públicos subnacionales. Por estas dos razones, las transferencias intergubernamentales tienen también la finalidad de compensar los desequilibrios fiscales horizontales (entre gobiernos subnacionales). 
Para analizar la existencia y la intensidad de las políticas distributivas regionales en el marco de las finanzas públicas subnacionales, se utilizan modelos simples de elasticidades de ingresos y gastos de los GSN respecto al Producto Bruto Geográfico (PBG) como proxy del ingreso; en estos modelos simples se supone que la diferencia entre los gastos y los recursos propios se cubren con transferencias. El test empírico consiste en comparar la elasticidad-ingreso del gasto público de los GSN, con la correspondiente elasticidad-ingreso de sus recursos propios (Bahl \& Wallace, 2003). Si los bienes provistos por los GSN son normales o superiores, la elasticidad-ingreso del gasto público será positiva. En general, también será positiva la elasticidad-ingreso de los recursos propios. La comparación de las elasticidades-ingreso y gasto provee un test sobre la existencia de políticas de igualación: si la elasticidad-ingreso del gasto es menor que la de los recursos propios es un indicador de existencia de un mecanismo de igualación fiscal regional. O sea, en los GSN con mayor ingreso per cápita, el gasto aumenta menos que sus recursos propios, ya que reciben menos transferencias que aquellos de menor ingreso per cápita. La diferencia entre las elasticidades-ingreso de los gastos y los recursos indica la intensidad de la política de igualación regional.

En un segundo ejercicio, con el fin de analizar el régimen de transferencias y observar su monto total y distribución entre jurisdicciones, se calcula la relación entre la transferencia recibida por cada GSN (el destino) y el aporte realizado para financiarlas (el origen).
Políticas fiscales alternativas. Impacto regional en distintos escenarios de descentralización y autonomía fiscal

Desde la perspectiva de las finanzas públicas comparadas, es posible analizar las siguientes cuestiones: i) si existen diferencias en cuanto a la disponibilidad de bienes públicos entre las jurisdicciones que se traduzcan en desigualdad territorial de oportunidades; ii) cuáles son las diferencias de recursos propios (debidas a diferentes bases imponibles, alícuotas y esfuerzo de administración tributaria) y en cuánto limitan el nivel de gastos; iii) si las transferencias compensan y en qué medida las diferencias de recursos propios; y iv) cuál es la correspondencia fiscal de cada jurisdicción. Para responder a estas preguntas se requiere construir escenarios cuyos resultados son útiles para el diseño, evaluación e instrumentación de políticas públicas.

En el escenario de autonomía total, cada gobierno debe igualar el gasto total con sus recursos propios. En este caso, en presencia de disparidades regionales de ingresos, se producen distintas disponibilidades de bienes públicos subnacionales -medidos con el gasto público- en cada jurisdicción y/o diferencias en los niveles de presión tributaria. La Figura 1 ilustra esta situación. En el eje vertical se mide el gasto per cápita y en el eje horizontal el ingreso per cápita. Si la alícuota es t0 y no hay transferencias intergubernamentales la jurisdicción rica $(R)$ tendrá un gasto per cápita superior al de la región pobre $(P)$ - cantidades GpcR y GpcP. Si la jurisdicción $\mathrm{P}$ desea obtener el mismo nivel de gasto que la región $R(\mathrm{GpcR})$ debe aumentar la presión tributaria hasta $t^{\prime \prime}>t_{0}$. Esto resulta en un trato diferente a las per- 
sonas según la jurisdicción en la que vivan. El escenario de autonomía total puede ser defendido porque incentiva la asignación eficiente de recursos (Bird, 2001): las jurisdicciones que reciben el beneficio del gasto cargan con el costo y la responsabilidad de gastar coincide con la de recaudar. El principal problema se origina en el hecho de que, como las regiones difieren en su capacidad tributaria, las ricas dispondrán de más bienes públicos que las pobres con la misma presión tributaria, o menor presión tributaria para obtener un nivel dado de gasto público.

Por esta razón la autonomía total no es un objetivo aceptado en forma pura. En las teorías de segunda generación del federalismo fiscal se propone que la igualdad entre los recursos propios y los gastos sea el objetivo de política para las jurisdicciones más ricas; para el resto de las jurisdicciones serán necesarias transferencias de igualación (Bird, 2010). Un punto interesante en este enfoque es que hay jurisdicciones, las más ricas, que no reciben transferencias (como sucede, p.ej. en Alemania y en el caso de las municipalidades en Chile). En la mayoría de los regímenes vigentes en el mundo todas las jurisdicciones reciben transferencias.

Figura 1. Incidencia regional de distintos escenarios de descentralización y autonomía fiscal

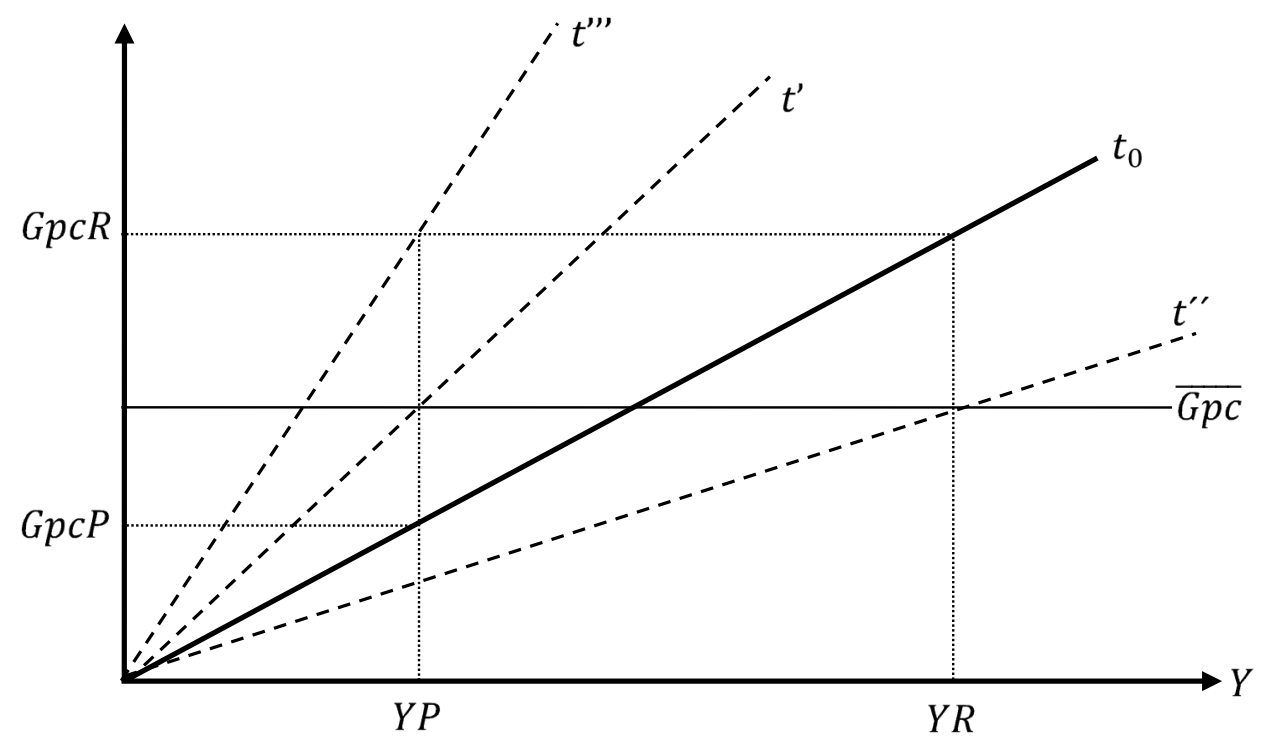


Una variante del caso anterior es la política de igualdad de la presión tributaria que se fundamenta en la necesidad de evitar la competencia tributaria (Break, 1967; Genschel \& Schwartz, 2011). Se supone que la base imponible en todas las jurisdicciones depende negativamente de su alícuota y positivamente de la alícuota del resto. Para atraer base imponible una jurisdicción disminuye su alícuota; lo mismo hará el resto de las jurisdicciones. El resultado será menor recaudación en todas y, por consiguiente, una tendencia a una provisión subóptima de bienes. En Tiebout (1956), en cambio, se visualiza la competencia interjurisdiccional como forma de alcanzar una solución eficiente. Desde otro punto de vista, la competencia tributaria es alentada por Brennan y Buchanan (1977) como medio de control del tamaño del gasto.

Una alternativa resulta cuando las dos jurisdicciones deben igualar la provisión de bienes públicos per cápita en un nivel fijado por el gobierno central $(\overline{G p c})$ con el objetivo de igualar oportunidades (escenario de igualdad de gasto per cápita). Si no existen transferencias intergubernamentales la jurisdicción $\mathrm{P}$ debe fijar una alícuota mayor que la jurisdicción R (t'vs t" en la Figura 1). En este caso, la igualdad de gasto público per cápita se logra al costo de un diferente trato impositivo en cada jurisdicción. El objetivo de igualdad de gasto per cápita en todas las jurisdicciones se fundamenta en que todas ellas dispongan de una canasta de bienes básicos o primarios. Una cuestión importante en este caso es seleccionar los bienes que componen esa canasta (Oakland, 1994); p. ej., la atención básica de la salud y la educación básica son bienes que todos los habitantes deberían recibir con in- dependencia de su lugar de residencia. La variedad de bienes aumenta con el tamaño de la población que permite cubrir los costos fijos y variables (es el efecto "zoo" de Oates, 1988).

Finalmente se supone un escenario de centralización tributaria en el que el gasto es financiado sólo con transferencias (los RP son iguales a cero). La política de financiar los gastos subnacionales totalmente con transferencias se fundamenta en que los costos de eficiencia de los tributos subnacionales son mayores que los de los nacionales debido a la mayor movilidad de las bases tributarias a nivel subnacional (Brueckner, 2004). Con la centralización tributaria total se evitarían esos costos, pero aparecerían los problemas de generar incentivos para la irresponsabilidad fiscal de los gobiernos subnacionales y de control macroeconómico (debido a la restricción presupuestaria blanda).

\section{CUANTIFICACIONES Y ESTIMACIONES}

\section{Descentralización Fiscal}

La teoría fiscal se desarrolló, desde sus comienzos y por largo tiempo, en contextos de gobiernos unitarios. La pregunta más importante siempre fue y es quién, entre el gobierno y el sector privado, hace qué; o sea, la línea divisoria entre dos mecanismos de asignación de recursos, el mercado y la planificación. El indicador empírico utilizado para medir la importancia relativa del sector público es el gasto público (y/o los recursos públicos) normalizado por la población y/o por el Producto Interno Bruto (PIB) de la economía. Una extensa literatura se dedicó a explicar esa relación y aparecieron en es- 
cena, entre otros factores, la ley de Wagner, la enfermedad de los costos de Baumol, la complementariedad o sustitución entre los bienes, y la estructura de edades de la población. Estos factores solo explicaron una parte del tamaño relativo del sector público lo que llevó a incorporar variables políticas e institucionales. En una segunda etapa se dejó de considerar al sector público como una estructura monolítica y, dado un tamaño del sector consolidado con relación al PIB, se incluyeron preguntas relacionadas con la estructura vertical: ¿Quién hace qué?, ¿Quién recauda qué?, ¿Cómo se coordinan las actividades? En términos de medición la variable anterior se complementó con la relación entre el gasto (recursos) público subnacional y el gasto público (recursos públicos) consolidado, y se estudiaron empíricamente los determinantes de la descentralización. En una tercera etapa, a las medidas anteriores se agregó la relación entre el gasto (recursos) público municipal y el gasto (recursos) público subnacional, o sea, la descentralización dentro del sector subnacional. El impulso para la mayor atención prestada a los gobiernos municipales vino dado por las demandas de democratización, para escapar al centralismo en las decisiones políticas y fiscales. En esta Sección se cuantifican los indicadores de descentralización fiscal y se los relacionan con variables demográficas y económicas. A tal fin, se utilizan datos fiscales de estados y municipios para el periodo 2000-2012, tomados de la ejecución presupuestaria publicada del Tesoro Nacional del Ministerio de Hacienda (www.tesouro.fazenda.gov.br/), el Portal Meu municipio (https:// meumunicipio.org.br) y Banco Interamericano de Desarrollo (BID). También se recurre a datos de población y producto bruto geo- gráfico publicado por el Instituto Brasileño de Geografía y Estadística. Los recursos propios de cada estado o municipio se definen como todos los recursos corrientes y de capital recaudados por dicha jurisdicción, comprendiendo recursos tributarios, (impuestos y tasas y contribuciones por mejoras), contribuciones sociales y económicas, recursos patrimoniales, agropecuarios, industriales y de servicios y otros no tributarios (multas y otros conceptos), más recursos de capital, sin considerar ingresos por uso del crédito. Las transferencias recibidas por estados y municipios se consideran como el total de transferencias percibidas (de la Unión, los estados y las multigubernamentales) deduciendo las afectaciones para FUNDEB. Por su parte, los gastos se consideran netos de las amortizaciones de la deuda.

\section{i) Descentralización de los gastos y los in- gresos públicos}

Las unidades del sector público de Brasil se representan como una pirámide con un gobierno central en la cúspide, seguido de 26 gobiernos estatales y más de 5.500 municipalidades en la base. La división de gastos y recursos entre esos niveles de gobierno luce como una pirámide invertida, especialmente para los recursos propios $(68,8 \%$ del total en cabeza del gobierno central, $25 \%$ los estados y $6,1 \%$ las municipalidades); la distribución de los gastos es $48,5 \%, 28,7 \%$ y 22,9 $\%$ respectivamente. La estructura del sector subnacional (variables fiscales municipales/ variables subnacionales) muestra a las municipalidades participando con el $44 \%$ de los gastos y con el 19,6\% de los recursos (Tabla 1, año 2012). 
Tabla 1. Estructura fiscal vertical de Brasil 2012

\begin{tabular}{ccccccccc}
\hline \multirow{2}{*}{ Nivel de gobierno } & \multicolumn{2}{c}{ En millones de \$R } & \multicolumn{2}{c}{ Part. \% } & \multicolumn{2}{c}{ En \% del PIB } & \multicolumn{2}{c}{ En \$R per cápita } \\
& Recursos & Gastos & Recursos & Gastos & Recursos & Gastos & Recursos & Gastos \\
\hline Federal & $1,188,952$ & 827,432 & 68.8 & 48.5 & 27.1 & 18.8 & 5,967 & 4,153 \\
Estadual & 432,466 & 489,185 & 25.0 & 28.7 & 9.8 & 11.1 & 2,171 & 2,455 \\
Municipal & 105,727 & 390,058 & 6.1 & 22.9 & 2.4 & 8.9 & 531 & 1,958 \\
Total & $1,727,145$ & $1,706,674$ & 100.0 & 100.0 & 39.3 & 38.9 & 8,669 & 8,566 \\
\hline
\end{tabular}

Fuente: elaboración propia en base a datos oficiales.

La evolución de los indicadores de descentralización revela un crecimiento de la participación de los gastos subnacionales. En particular, es notable el crecimiento de la participación de los gastos a nivel municipal. La mayor participación de los gastos municipales en el consolidado no ha sido acompañada de mayores recursos propios. Esto hace a las finanzas locales más dependientes de transferencias de los niveles superio-

ii) Grado de descentralización del sector subnacional por Estados.

Los estados no son un conjunto homogéneo en cuanto a su estructura vertical (Grin \& Abruzzo, 2016). Las heterogeneidades son muy marcadas a nivel subnacional, tal como se resume en la Tabla 2. res de gobierno.

Tabla 2. Gasto y recursos per cápita en los GSN intermedios, municipios y al nivel subnacional (2012). Promedio=100

\begin{tabular}{cccccccc}
\hline $\begin{array}{c}\text { Nivel de gobi- } \\
\text { erno }\end{array}$ & $\begin{array}{c}\text { Variable } \\
\text { fiscal }\end{array}$ & Mínimo & Media & Máximo & Máx/Min & $\begin{array}{c}\text { Desvío } \\
\text { estándar }\end{array}$ & $\begin{array}{c}\text { Coeficiente } \\
\text { de variación }\end{array}$ \\
\hline Estados & & 54 & 100 & 194 & 3.6 & 41 & 0.41 \\
Municipal & Gastos & 62 & 100 & 129 & 2.1 & 21 & 0.21 \\
Subnacional & & 61 & 100 & 158 & 2.6 & 26 & 0.26 \\
Estados & & 72 & 100 & 206 & 2.8 & 39 & 0.39 \\
Municipal & Recursos & 28 & 100 & 185 & 6.5 & 41 & 0.41 \\
Subnacional & propios & 35 & 100 & 165 & 4.7 & 36 & 0.36 \\
\hline
\end{tabular}

Una pregunta de interés es en qué medida la teoría de la descentralización fiscal puede explicar estas diferencias en el sector subnacional de gobierno. Partiendo del teorema de la descentralización de Oates (1977) y de los desarrollos de Oates (1985) y Wallis y Oates (1988), se indaga este punto. Se pre- sentan estimaciones cross-section para cuatro años seleccionados (2003, 2006, 2009 y 2012 , con 26 observaciones en cada uno) y para el pool de los trece años (338 observaciones), suponiendo que los coeficientes son homogéneos entre jurisdicciones y a lo largo del tiempo. El test de Ramsey sugiere 
que en algunas de estas estimaciones hay variables omitidas, razón por la que se estimó un modelo de efectos fijos para captar la influencia de variables no observables (Tabla 3). Los comentarios en el texto se refieren a los resultados del modelo de efectos fijos salvo cuando se aclare lo contrario. La descentralización tanto de gastos como de recursos propios es mayor en los Estados con mayor población: la mayor importancia relativa de las municipalidades en los estados más poblados permite adaptar mejor la oferta a la demanda de bienes municipales a la vez que el costo se reparte en un grupo de mayor tamaño (economías de escala). El coeficiente del PBGpc es positivo y significativo para los gastos y los recursos propios. Los resultados se mantienen en las estimaciones cross-section excepto para la elasticidad gastos-PBG.

Tabla 3. Elasticidades estimadas para descentralización subnacional de gastos y recursos

\begin{tabular}{|c|c|c|c|c|c|c|c|}
\hline $\begin{array}{c}\text { Variable } \\
\text { endógena }\end{array}$ & $\begin{array}{l}\text { Elasticidad } \\
\text { estimada }\end{array}$ & 2003 & 2006 & 2009 & 2012 & $\begin{array}{l}\text { Coef. Homo- } \\
\text { géneos }\end{array}$ & Efectos fijos \\
\hline \multirow{2}{*}{$\begin{array}{c}\text { Descentralización subnacio- } \\
\text { nal de gastos }\end{array}$} & Población & $0.192^{* \star *}$ & $0.185^{\star * *}$ & $0.185^{\star * *}$ & $0.200^{* * *}$ & $0.174^{* * *}$ & \multirow[t]{3}{*}{$0.558^{\star * \star}$} \\
\hline & $-0.110^{*}$ & $-0.150^{* *}$ & $-0.147^{* *}$ & -0.097 & -0.013 & $0.073^{* * *}$ & \\
\hline PBGpc & -0.026 & -0.003 & -0.023 & $-0.038^{*}$ & $-0.021^{* * *}$ & & \\
\hline \multicolumn{8}{|l|}{ Superficie } \\
\hline \multirow{2}{*}{$\begin{array}{l}\text { Descentralización subnacio- } \\
\text { nal de recursos }\end{array}$} & Población & $0.161^{* * *}$ & $0.140^{* * *}$ & $0.134^{\star * *}$ & $0.105^{* * *}$ & $0.139^{* * *}$ & \multirow[t]{2}{*}{$0.679^{\star *}$} \\
\hline & 0.093 & 0.164 & $0.120^{\star *}$ & $0.163^{* * *}$ & $0.134^{* * *}$ & $0.115^{\star *}$ & \\
\hline \multicolumn{8}{|l|}{ PBGpc } \\
\hline Superficie & -0.039 & -0.052 & $-0.061^{* *}$ & $-0.072^{* * *}$ & $-0.052^{* * *}$ & & \\
\hline Nro. De observaciones & & 26 & 26 & 26 & 26 & 338 & 338 \\
\hline
\end{tabular}

${ }^{*} p<.1 ;{ }^{* *} p<.05 ;{ }^{* * *} p<.01$

Estos resultados son importantes para la política fiscal hacia el futuro. Si se prevé que las municipalidades tendrán una participación creciente dentro del sector público subnacional, la modernización y fortalecimiento municipal debería ocupar un lugar importante en la agenda de las políticas públicas. Algunos temas de esta agenda comprenden: i) El rediseño del financiamiento con recursos propios. La literatura sobre asignación de fuentes tributarias a cada nivel de gobierno brinda un conjunto de guías que deberían tenerse en cuenta en el rediseño (p.ej. Bird, 2010). Una recomendación de Bird es que para los gobiernos locales el principal impuesto es sobre la propiedad inmueble, complementado con un impuesto bien diseñado sobre las actividades económicas. Cabe agregar los impuestos sobre vehículos. Las teorías de segunda generación del federalismo aportan también una nueva visión al considerar que si el gobierno municipal se financia mayoritariamente con recursos propios tendrá incentivos para promover del desarrollo local ya que aumentará la base imponible municipal (capacidad tributaria); ii) El rediseño de los sistemas de transferencias (evitando crear incentivos que desalienten 
el esfuerzo fiscal propio y/o que alienten la expansión excesiva del gasto). Las teorías de segunda generación consideran que las transferencias originan una restricción presupuestaria blanda que lleva a comportamiento fiscales no prudentes, razón por la cual proponen una visión de la asignación de recursos y gastos a los niveles subnacionales de gobierno distinta a la de las teorías de primera generación: primero determinar los gastos y luego asignar los recursos de modo de generar una restricción presupuestaria fuerte. Bird (2010) propone que las regiones más ricas se autofinancien; iii) La mejora en la eficiencia (reducción de costos) en la prestación de los servicios. Este es un campo importante y con relativamente pocos avances debido a las dificultades, sobre todo de tipo informativo. Entre las preguntas importantes a las que deberían dirigirse los esfuerzos están: qué relación existe entre necesidad de gasto y gasto per cápita; qué relación con las transferencias per cápita; qué relación existe entre los recursos propios y la capacidad tributaria.

Como parte del análisis econométrico, se realizó la estimación de los gastos y recursos propios per cápita a nivel subnacional con la población y el producto bruto per cápita (Tabla 4). Los resultados de estas esti- maciones revelan que el gasto per cápita de los Estados, las municipalidades y el agregado subnacional aumentan con el tamaño de la población, siendo mayor el coeficiente correspondiente a las municipalidades. En consecuencia, a medida que aumenta la población aumenta la descentralización subnacional. Algo similar ocurre con los recursos propios. Resumiendo, las participaciones de las municipalidades en el gasto subnacional y en los recursos propios aumentan con el tamaño de la población. Los gastos y recursos propios de los estados, municipalidades $y$ el total subnacional, se relacionan positivamente con el PBGpc, indicando que se trata de bienes "normales" y que los recursos propios aumentan con el PBGpc. Como ocurría con la población, los coeficientes de las municipalidades son mayores que los de los Estados de modo que la descentralización subnacional aumenta con el tamaño del PBG. Estos resultados indican, tal como resulta de la Tabla 4 que la descentralización fiscal subnacional es mayor en los Estados con mayor población y mayor PBG per cápita. Carvalho de Andrada Lima y Silveira Neto (2018) estudian el impacto de la creación de municipalidades en Brasil desde 1991 y concluyen que ha originado pérdida de economías de escala a lo que se agregan los comportamientos de rent-seeking. 
Alberto Porto - Walter Rubén Rosales

Tabla 4. Elasticidades estimadas para gastos y recursos propios, en términos per cápita

\begin{tabular}{|c|c|c|c|c|c|c|c|c|}
\hline Variable endógena & $\begin{array}{l}\text { Nivel de } \\
\text { gobierno }\end{array}$ & $\begin{array}{l}\text { Elasticidad } \\
\text { estimada }\end{array}$ & 2003 & 2006 & 2009 & 2012 & $\begin{array}{l}\text { Coef. Ho- } \\
\text { mogéneos }\end{array}$ & Efectos fijos \\
\hline \multirow{9}{*}{ Gastos per cápita } & \multirow{3}{*}{ Estadual } & Población & $-0.199^{* * *}$ & $-0.241^{* * *}$ & $-0.252^{* * *}$ & $-0.226^{* * *}$ & $-0.243^{* * *}$ & $0.919^{* * *}$ \\
\hline & & PBGpc & $0.603^{* * *}$ & $0.593^{* * *}$ & $0.593^{* * *}$ & $0.581^{* * *}$ & $0.824^{* * *}$ & $0.994^{* * *}$ \\
\hline & & Superficie & 0.020 & 0.018 & 0.030 & 0.040 & $0.038^{\star * *}$ & \\
\hline & \multirow{3}{*}{ Municipal } & Población & 0.040 & 0.003 & 0.010 & 0.040 & -0.023 & $1.586^{\star \star *}$ \\
\hline & & PBGpc & $0.450^{\star \star \star}$ & $0.371^{* * *}$ & $0.349^{* * *}$ & $0.427^{\star * *}$ & $0.845^{\star * *}$ & $1.137^{\star \star *}$ \\
\hline & & Superficie & $-0.047^{\star \star \star}$ & -0.017 & $-0.035^{\star}$ & $-0.058^{\star * *}$ & $-0.044^{* * *}$ & \\
\hline & \multirow{3}{*}{ Subnacional } & Población & $-0.151^{* * *}$ & $-0.182^{\star * *}$ & $-0.174^{\star \star \star}$ & $-0.160^{\star * \star}$ & $-0.197^{* * *}$ & $1.028^{\star * *}$ \\
\hline & & PBGpc & $0.560^{* \star *}$ & $0.521^{* * *}$ & $0.495^{\star * *}$ & $0.524^{* * *}$ & $0.858^{* * *}$ & $1.064^{* * *}$ \\
\hline & & Superficie & -0.020 & -0.013 & -0.012 & -0.020 & $-0.023^{* *}$ & \\
\hline \multirow{9}{*}{$\begin{array}{l}\text { Recursos propios per } \\
\text { cápita }\end{array}$} & \multirow{3}{*}{ Estadual } & Población & 0.055 & -0.003 & 0.012 & -0.020 & 0.008 & $2.378^{\star * *}$ \\
\hline & & PBGpc & $0.942^{\star \star \star}$ & $0.869^{* \star *}$ & $0.864^{\star * *}$ & $0.883^{\star * *}$ & $1.031^{* * *}$ & $0.898^{\star \star *}$ \\
\hline & & Superficie & 0.008 & 0.012 & 0.010 & $0.039^{*}$ & $0.030^{* * *}$ & \\
\hline & \multirow{3}{*}{ Municipal } & Población & $0.233^{\star * *}$ & $0.156^{\star * \star}$ & $0.162^{\star \star *}$ & $0.101^{* *}$ & $0.161^{\star \star \star}$ & $3.204^{\star * *}$ \\
\hline & & PBGpc & $1.127^{* \star \star}$ & $1.105^{\star * *}$ & $1.077^{* \star *}$ & $1.127^{\star \star \star}$ & $1.252^{\star * \star}$ & $1.027^{\star \star \star}$ \\
\hline & & Superficie & $-0.063^{\star *}$ & $-0.060^{* *}$ & $-0.081^{* * *}$ & $-0.060^{* * *}$ & $-0.068^{* * *}$ & \\
\hline & \multirow{3}{*}{ Subnacional } & Población & 0.072 & 0.016 & 0.028 & -0.004 & $0.022^{*}$ & $2.525^{\star \star \star}$ \\
\hline & & PBGpc & $1.035^{\star \star *}$ & $0.941^{* * *}$ & $0.957^{\star * *}$ & $0.964^{* * *}$ & $1.117^{\star \star *}$ & $0.912^{* \star *}$ \\
\hline & & Superficie & -0.024 & -0.008 & -0.020 & 0.012 & $-0.015^{\star}$ & $0.919^{\star * *}$ \\
\hline \multicolumn{2}{|c|}{ Nro. De observaciones } & & 26 & 26 & 26 & 26 & 338 & 338 \\
\hline
\end{tabular}

${ }^{*} p<.1 ;{ }^{* *} p<.05 ;{ }^{* * *} p<.01$

\section{Autonomía fiscal subnacional}

A nivel agregado (Estados más municipalidades) Brasil registra un $61 \%$ de correspondencia fiscal (autonomía) para el año
2012; para los Estados es del $72,8 \%$ y para las municipalidades del $27 \%$ (Tabla 5). Hay margen para mejorar la correspondencia entre ingresos y gastos (la "conexión Wickselliana”; Bird \& Slack, 2014).

Tabla 5. Correspondencia fiscal. Por nivel de gobierno (en porcentaje)

\begin{tabular}{cccccccccccccc}
\hline Nivel de gobierno & $\mathbf{2 0 0 2}$ & $\mathbf{2 0 0 3}$ & $\mathbf{2 0 0 4}$ & $\mathbf{2 0 0 5}$ & $\mathbf{2 0 0 6}$ & $\mathbf{2 0 0 7}$ & $\mathbf{2 0 0 8}$ & $\mathbf{2 0 0 9}$ & $\mathbf{2 0 1 0}$ & $\mathbf{2 0 1 1}$ & $\mathbf{2 0 1 2}$ & Promedio \\
\hline Estadual & 74.0 & 78.0 & 79.9 & 79.0 & 77.4 & 77.9 & 75.0 & 72.9 & 73.1 & 74.0 & 72.8 & 75.8 \\
Municipal & 30.9 & 30.8 & 28.5 & 29.2 & 27.3 & 27.4 & 25.9 & 25.6 & 26.1 & 26.4 & 27.1 & 27.7 \\
Agregado subnacional & 67.4 & 69.8 & 69.0 & 69.8 & 65.9 & 65.7 & 63.2 & 60.8 & 61.2 & 61.6 & 61.2 & 65.1 \\
\hline
\end{tabular}


La correspondencia fiscal varía notablemente entre los estados y los municipios. La Tabla 6 presenta la correspondencia fiscal normalizada (promedio=100) para el año 2012. Hay Estados con correspondencia fiscal 65
$\%$ inferior al promedio y otros con valor $38 \%$ superior; municipalidades entre $60 \%$ y 20 $\%$, respectivamente; para el agregado subnacional entre el $59 \%$ inferior al promedio y $20 \%$ superior.

Tabla 6. Correspondencia fiscal estadual, municipal y subnacional por país (2012). Promedio $=100$

\begin{tabular}{lcccccc}
\hline Nivel de gobierno & Mínimo & Media & Máximo & Máx/Min & $\begin{array}{c}\text { Desvío } \\
\text { estándar }\end{array}$ & Coeficiente de variación \\
\hline Estadual & 35 & 100 & 138 & 4.0 & 28 & 0.28 \\
Municipal & 40 & 100 & 144 & 3.6 & 26 & 0.26 \\
Subnacional & 41 & 100 & 120 & 3.0 & 25 & 0.25 \\
\hline
\end{tabular}

La correspondencia fiscal aumenta con la población a nivel subnacional, municipal y estadual: Las jurisdicciones más pobladas dependen menos de las transferencias. Los coeficientes del PBGpc son negativos indi- cando menor correspondencia fiscal en las jurisdicciones en las que el producto per cápita es mayor -explicado por las diferentes elasticidades de los ingresos y los gastos (Tabla 7).

Tabla 7. Elasticidades estimadas para la correspondencia fiscal

\begin{tabular}{cccccccc}
\hline Nivel de gobierno & $\begin{array}{c}\text { Elasticidad } \\
\text { estimada }\end{array}$ & 2003 & 2006 & 2009 & 2012 & $\begin{array}{c}\text { Coef. Homo- } \\
\text { géneos }\end{array}$ & Efectos fijos \\
\hline \multirow{3}{*}{ Estadual } & Población & $0.255^{* * *}$ & $0.238^{* * *}$ & $0.263^{* * *}$ & $0.206^{* * *}$ & $0.251^{* * *}$ & $1.459^{* * *}$ \\
& PBGpc & $0.339^{* * *}$ & $0.276^{* * *}$ & $0.271^{* * *}$ & $0.303^{* * *}$ & $0.207^{* * *}$ & $-0.096^{* *}$ \\
& Superficie & -0.012 & -0.006 & -0.020 & -0.001 & -0.008 & \\
Municipal & Población & $0.192^{* * *}$ & $0.153^{* * *}$ & $0.151^{* * *}$ & $0.062^{* *}$ & $0.184^{* * *}$ & $1.618^{* * *}$ \\
& PBGpc & $0.678^{* * *}$ & $0.734^{* * *}$ & $0.728^{* * *}$ & $0.700^{* * *}$ & $0.407^{* * *}$ & $-0.110^{* *}$ \\
& Superficie & -0.016 & $-0.043^{* *}$ & $-0.046^{* *}$ & -0.002 & $-0.024^{* *}$ & $1.498^{* * *}$ \\
Subnacional & Población & $0.223^{* * *}$ & $0.198^{* * *}$ & $0.202^{* * *}$ & $0.156^{* * *}$ & $0.219^{* * *}$ & $-0.153^{* * *}$ \\
& PBGpc & $0.475^{* * *}$ & $0.420^{* * *}$ & $0.461^{* * *}$ & $0.440^{* * *}$ & $0.259^{* * *}$ & $1.459^{* * *}$ \\
\hline
\end{tabular}

${ }^{*} p<.1 ;{ }^{* *} p<.05 ;{ }^{* * *} p<.01$ 


\section{Aspectos distributivos regionales. Cuan- tificación de las disparidades fiscales ho- rizontales}

En esta sección se presentan los resultados de la cuantificación de las disparidades horizontales de los gastos, ingresos y transferencias.

Disparidades horizontales de gastos e ingresos en el nivel subnacional de gobierno

Se observan grandes disparidades horizontales del gasto per cápita en los Estados, municipalidades y en el agregado subnacional. La Tabla 3 muestra estas disparidades utilizando índices con relación al promedio nacional igual a cien. En los Estados se verifica que la relación entre los valores máximo y mínimo del gasto per cápita es de 3,6 veces. En los gobiernos municipales, la relación es de 2,1 veces. Estos resultados son importantes al evaluar la descentralización: el gasto público per cápita varía entre un 54 $\%$ menor al promedio y un $94 \%$ superior al promedio a nivel de departamentos. Las diferencias son menores a nivel de municipalidades: entre $38 \%$ inferior al promedio y $29 \%$ superior. Una conclusión preliminar es que hay fuertes disparidades en el nivel de gastos per cápita, lo que puede originar desigualdad de oportunidades en cuanto al acceso a los bienes públicos subnacionales. Cabe aclarar que las diferencias en los gastos per cápita pueden resultar de diferencias en los costos de provisión y/o en la calidad de los bienes

También del lado de los recursos propios per cápita existen importantes disparidades medidas con relación al promedio (Tabla 3). A nivel de los Estados los recursos propios varían entre un $28 \%$ inferior al promedio y un $106 \%$ superior; en las municipalidades entre $72 \%$ inferior y $85 \%$ superior. La conclusión es que no existe igualdad en la generación de recursos propios per cápita, de modo que se requieren las transferencias de igualación desde niveles superiores de gobierno para lograr niveles similares de gasto per cápita.

\section{Transferencias intergubernamentales}

Las transferencias son el medio para compensar los desequilibrios fiscales horizontales. La Tabla 8 muestra las transferencias en términos per cápita recibidas por los Estados, las municipalidades y el agregado subnacional para el año 2012. En los Estados varían entre $52 \%$ inferior al promedio y 441 $\%$ superior; en las municipalidades entre 31 $\%$ inferior y $31 \%$ superior y para el agregado subnacional entre $33 \%$ inferior y $204 \%$ superior. 
DESCENTRALIZACIÓN SUBNACIONAL, AUTONOMÍA Y DISPARIDADES FISCALES EN BRASIL

Tabla 8. Transferencias per cápita a GSN estadual, municipios y al nivel subnacional (2012). Promedio $=100$

\begin{tabular}{ccccccc}
\hline Nivel de gobierno & Mínimo & Media & Máximo & Máx/Min & Desvío estándar & Coeficiente de variación \\
\hline Estadual & 48 & 100 & 541 & 11.3 & 541 & 5.41 \\
Municipal & 69 & 100 & 131 & 1.9 & 15 & 0.15 \\
Subnacional & 67 & 100 & 304 & 4.5 & 62 & 0.62 \\
\hline
\end{tabular}

Dos preguntas de importancia relativas a las disparidades fiscales horizontales son: si existe igualdad de oportunidades entre los GSN en cuanto a la disponibilidad de bienes públicos (medidos con el gasto público) y si los recursos propios son similares entre gobiernos. Las respuestas han sido negativas. Una pregunta adicional que resulta de estos hallazgos es si las transferencias de los niveles centrales de gobierno compensan o amplifican las diferencias de recursos propios.

Relación entre transferencias intergubernamentales, población y producto bruto per cápita.

En Brasil existen transferencias entre la Unión y los Estados, la Unión y las Municipalidades y los Estados y las Municipalidades. La transferencia más importante para los Estados es el FPE (Fondo de participación de los Estados) que distribuye el 21,5 $\%$ del impuesto a la Renta (IR) y el impuesto a los productos industrializados (IPI); se distribuye en base a coeficientes fijos que reflejan criterios redistributivos. Las otras transferencias importantes son del FUNDEB y para pago de salarios en educación. Estas tres transferencias dan cuenta del 80 $\%$ del total. De la Unión a las Municipalidades el más importante es el Fondo de Participación Municipal que distribuye el 23,5 $\%$ del (IR) y del IPI; se distribuye por tipos de municipios y la variable más importante es la población. El FUNDEB es la segunda transferencia en importancia. Estas dos transferencias representan el $88 \%$ del total. Entre los Estados y las Municipalidades la transferencia más importante es el ICMS: $25 \%$ de lo recaudado, $75 \%$ devolutivo y 25 $\%$ redistributivo y el FUNDEB. Entre las dos representan el $72 \%$ del total de transferencias (para un análisis detallado ver Villela, Afonso y Calvo (2016). Escapa al objetivo de este trabajo el análisis en particular de cada uno de estos mecanismos de transferencia. En el análisis econométrico se utilizan como variables explicativas la población, la superficie y el PBGpc que se consideran representativos de las variables utilizadas como distribuidores (la población como indicador de demanda de bienes, la superficie indicadora de costos y el PBGpc indicador del ingreso de la jurisdicción. El signo de cada variable es una cuestión empírica dado que un mismo distribuidor tiene distintos efectos según el tipo de transferencia; por ejemplo, un signo positivo para el PBGpc puede interpretarse indicando el criterio devolutivo, pero como también existen distribuidores redistributivos ese signo podría compensarse o más que compensarse. Algo similar ocurre con los signos de las otras dos variables.

Se estimaron las elasticidades de las transferencias per cápita con la población y el PBGpc. El coeficiente de la población es 
negativo para los Estados, positivo para las municipalidades y cero para el agregado (Tabla 9). Varios efectos que van en direcciones opuestas pueden explicar estos resultados: economías de escala (difusión de los costos fijos y variables), deseconomías de escala (costos de congestión, fragmentación), variedad de bienes (efecto "zoo" de Oates, 1988), y mejor aprovechamiento de la capacidad tributaria propia, entre otros. El coeficiente correspondiente al PBGpc es positivo para las tres jurisdicciones.

Tabla 9. Elasticidades estimadas para las transferencias per cápita.

\begin{tabular}{cccccccc}
\hline Nivel de gobierno & $\begin{array}{c}\text { Elasticidad } \\
\text { estimada }\end{array}$ & 2003 & 2006 & 2009 & 2012 & $\begin{array}{c}\text { Coef. Homo- } \\
\text { géneos }\end{array}$ & $\begin{array}{c}\text { Efectos } \\
\text { fijos }\end{array}$ \\
\hline \multirow{3}{*}{ Estadual } & Población & $-0.574^{* * *}$ & $-0.503^{* * *}$ & $-0.472^{* * *}$ & $-0.402^{* * *}$ & $-0.569^{* * *}$ & $-1.643^{* * *}$ \\
& PBGpc & $-0.248^{*}$ & $-0.295^{* *}$ & -0.050 & -0.169 & $0.365^{* * *}$ & $1.350^{* * *}$ \\
& Superficie & -0.002 & -0.033 & 0.003 & 0.013 & 0.034 & \\
Municipal & Población & -0.037 & $-0.076^{*}$ & -0.054 & -0.009 & $-0.090^{* * *}$ & $1.650^{* * *}$ \\
& PBGpc & $0.257^{* * *}$ & $0.211^{* * *}$ & $0.188^{* * *}$ & $0.228^{* * *}$ & $0.716^{* * *}$ & $1.048^{* * *}$ \\
& Superficie & 0.001 & 0.000 & -0.014 & $-0.041^{* *}$ & -0.025 & \\
Subnacional & Población & $-0.334^{* * *}$ & $-0.338^{* * *}$ & $-0.324^{* * *}$ & $-0.277^{* * *}$ & $-0.375^{* * *}$ & -0.027 \\
& PBGpc & -0.245 & -0.129 & -0.035 & 0.007 & $0.535^{* * *}$ & $1.219^{* * *}$ \\
Nro. De observaciones & Superficie & 0.043 & 0.001 & 0.007 & -0.052 & -0.011 & $-1.643^{* * *}$ \\
\hline
\end{tabular}

${ }^{*} p<.1 ;{ }^{* *} p<.05 ;{ }^{* * *} p<.01$

Transferencias e igualación fiscal

En la tabla 10 se comparan las elasticidades-PBG de los gastos y recursos propios como indicador de efecto igualador de las transferencias (Bahl \& Wallace, 2003). Las estimaciones cross section sugieren la presencia de ese efecto aunque no se confirma con el modelo más adecuado de Efectos fijos -en el que los coeficientes son mayores para los gastos. 
DESCENTRALIZACIÓN SUBNACIONAL, AUTONOMÍA Y DISPARIDADES FISCALES EN BRASIL

Tabla 10. Elasticidades ingreso estimadas por nivel de gobierno

\begin{tabular}{lcccccc}
\hline Variables endógenas & 2003 & 2006 & 2009 & 2012 & $\begin{array}{c}\text { Coef. Homogé- } \\
\text { neos }\end{array}$ & Efectos fijos \\
\hline Estadual & & & & & & \\
Gasto per cápita & $0.603^{* * *}$ & $0.593^{* * *}$ & $0.593^{* * *}$ & $0.581^{* * *}$ & $0.824^{* * *}$ & $0.994^{* * *}(2)$ \\
Recursos propios per cápita & $0.942^{* * *}$ & $0.869^{* * *}$ & $0.864^{* * *}$ & $0.883^{* * *}$ & $1.031^{* * *}$ & $0.898^{* * *}(1)$ \\
Municipal & & & & & & \\
Gasto per cápita & $0.450^{* * *}$ & $0.371^{* * *}$ & $0.349^{* * *}$ & $0.427^{* * *}$ & $0.845^{* * *}$ & $1.137^{* * *}(1)$ \\
Recursos propios per cápita & $1.127^{* * *}$ & $1.105^{* * *}$ & $1.077^{* * *}$ & $1.127^{* * *}$ & $1.252^{* * *}$ & $1.027^{* * *}(2)$ \\
Subnacional & & & & & & \\
Gasto per cápita & $0.560^{* * *}$ & $0.521^{* * *}$ & $0.495^{* * *}$ & $0.524^{* * *}$ & $0.858^{* * *}$ & $1.064^{* * *}(1)$ \\
Recursos propios per cápita & $1.035^{* * *}$ & $0.941^{* * *}$ & $0.957^{* * *}$ & $0.964^{* * *}$ & $1.117^{* * *}$ & $0.912^{* * *}(1)$ \\
Nro. De observaciones & 26 & 26 & 26 & 26 & 338 & 338 \\
\hline
\end{tabular}

(1) En base al test de Wald se rechaza la hipótesis nula de que el coeficiente es estadísticamente igual a 1 al 5 $\%$ de significatividad.

(2) En base al test de Wald no se rechaza la hipótesis nula de que el coeficiente es estadísticamente igual a 1 al $5 \%$ de significatividad.

Una forma más adecuada de cuantificar el efecto igualador resulta de calcular la diferencia para cada jurisdicción entre las transferencias recibidas y el aporte realizado para su financiamiento, o sea, qué jurisdicciones "ganan" y cuales "pierden". Se utilizaron tres criterios para estimar el aporte de cada jurisdicción: i) que los recursos destinados a transferencias se recaudan territorialmente como los recursos propios de los Estados y de las Municipalidades; ii) que la recaudación es proporcional al PBG de cada jurisdicción; iii) que la recaudación resulta del promedio de PBG, los RP de los estados y la población (1/3 de ponderación cada uno). Los resultados de las estimaciones del aporte neto se presentan en la Tabla 11 y revelan la existencia de un mecanismo de igualación en las transferencias: con los tres criterios, el aporte neto aumenta con la población y con el PBG tanto para los Estados como para el conjunto subnacional.

Tabla 11. Aporte neto por niveles de gobierno. Semi-elasticidad respecto de población y PBGpc (2012)

\begin{tabular}{|c|c|c|c|c|c|}
\hline Criterio & $\begin{array}{l}\text { Nivel de go- } \\
\text { bierno }\end{array}$ & Población & $\begin{array}{l}\text { PBG per } \\
\text { cápita }\end{array}$ & $\mathrm{R}^{2}$ & $\begin{array}{l}\text { Nro. } \\
\text { Obs. }\end{array}$ \\
\hline \multirow{3}{*}{$\begin{array}{l}\text { Aporte de cada nivel de gobierno distribuido en función } \\
\qquad \text { de recursos propios }\end{array}$} & Estadual & $662^{* * *}$ & $686^{* * *}$ & 0.604 & 27 \\
\hline & Municipal & $169^{* * *}$ & $767^{* \star *}$ & 0.800 & 26 \\
\hline & Subnacional & $778^{* * *}$ & $1517^{* * *}$ & 0.739 & 27 \\
\hline \multirow{3}{*}{$\begin{array}{l}\text { Aporte de cada nivel de gobierno distribuido en función } \\
\qquad \text { de PBG }\end{array}$} & Estadual & $671^{* * *}$ & $798^{* \star *}$ & 0.643 & 27 \\
\hline & Municipal & 59 & $756^{* * *}$ & 0.821 & 26 \\
\hline & Subnacional & $744^{* * *}$ & $1915^{* * *}$ & 0.739 & 27 \\
\hline \multirow{3}{*}{$\begin{array}{l}\text { Aporte de cada nivel de gobierno distribuido en función } \\
\text { de población, PBG y recursos propios }\end{array}$} & Estadual & $666^{* * *}$ & $544^{* *}$ & 0.596 & 27 \\
\hline & Municipal & $82^{*}$ & $403^{* * *}$ & 0.659 & 26 \\
\hline & Subnacional & $751^{\text {***}}$ & $1243^{* * *}$ & 0.693 & 27 \\
\hline
\end{tabular}


Alternativas de política. Impacto regional en distintos escenarios de descentralización y autonomía fiscal

Se calculan los efectos distributivos resultantes de diferentes políticas públicas de descentralización sobre las variables fiscales de los Estados, municipalidades y el agregado subnacional (Tabla 12). Se comparan los valores del índice de desigualdad de Gini (ponderado con población) resultantes para cada escenario de política con el comportamiento real observado. El ejercicio es simple ya que supone que ante un cambio de política, las variables se ajustan pasivamente según lo requerido por la política en cuestión. Los escenarios son:

(1) Autonomía total con el gasto igual a los recursos propios y transferencias iguales a cero. Este escenario permite observar lo que ocurriría con la distribución de los gastos, si existiese correspondencia fiscal media perfecta en todas las jurisdicciones. El resultado es un aumento en las disparidades de gasto per cápita. Para el agregado subnacional el coeficiente de Gini aumentaría de 0,14 a 0,24, para los Estados de 0,17 a 0,22 y para las municipalidades de 0,14 a 0,35. El resultado de este ejercicio era esperable dado que cada jurisdicción debe ajustar sus gastos a su restricción presupuestaria. El resultado revela que las transferencias cumplen un rol de nivelación de los gastos.

(2) Presión tributaria igual al promedio en todas las jurisdicciones, en cuyo caso el gasto es ajustado en función de las diferencias entre la presión tributaria de cada jurisdicción y la presión tributaria promedio, manteniendo las transferencias reales. A nivel agregado y de los departamentos aumentan entre uno $y$ tres puntos porcentuales las desigualdades de recaudación propia, gasto per cápita y correspondencia fiscal. El aumento de la desigualdad de los RP se debe a que, por el cambio de política tributaria, las jurisdicciones con mayor PBGpc aumentan la presión tributaria y las de menor PBGpc la disminuyen. A nivel de municipalidades disminuye significativamente el Gini de la recaudación propia (10 puntos) y el de la correspondencia fiscal (7 puntos); también disminuye el del gasto en dos puntos porcentuales. En este caso las municipalidades con mayor PBGpc disminuyen su presión tributaria para llegar al promedio que es el objetivo de política y las de menor PBGpc la aumentan. Estos resultados son compatibles con las elasticidades-PBG estimadas.

(3) Gasto per cápita igual al promedio en todas las jurisdicciones, manteniendo las transferencias reales y ajustando el presupuesto con variaciones de la presión tributaria. En este escenario se incrementa muy significativamente la desigualdad de la presión tributaria y disminuye también significativamente la diferencia de recaudación per cápita. Varias combinaciones de la situación fiscal y del PBG de las jurisdicciones pueden dar lugar a este resultado.

(4) Transferencias per cápita iguales al promedio, con el gasto financiado solo con transferencias. En este caso extremo, se logra la igualdad en todos los niveles de gobierno, pero con grandes diferencias en el nivel absoluto del gasto per cápita comparado con la situación real. 
DESCENTRALIZACIÓN SUBNACIONAL, AUTONOMÍA Y DISPARIDADES FISCALES EN BRASIL

Tabla 12. Indicador de GINI para distintas alternativas de distribución regional (2012)

\begin{tabular}{|c|c|c|c|c|}
\hline \multirow{2}{*}{ Estados } & \multirow{2}{*}{ Variables fiscales } & \multicolumn{3}{|c|}{ Brasil } \\
\hline & & Departamentos & Municipios & Nivel Subnacional \\
\hline \multirow{6}{*}{ Situación real } & Rec. propios per cápita & 0.22 & 0.35 & 0.24 \\
\hline & Transf. Per cápita & 0.18 & 0.07 & 0.10 \\
\hline & Presión tributaria & 0.06 & 0.12 & 0.05 \\
\hline & Gasto per cápita & 0.17 & 0.14 & 0.14 \\
\hline & Correspondencia fiscal & 0.11 & 0.23 & 0.13 \\
\hline & Rec. propios per cápita & 0.22 & 0.35 & 0.24 \\
\hline \multirow{4}{*}{ Autonomía total (1) } & Transf. Per cápita & & & \\
\hline & Presión tributaria & 0.06 & 0.12 & 0.05 \\
\hline & Gasto per cápita & 0.22 & 0.35 & 0.24 \\
\hline & Correspondencia fiscal & 0.00 & 0.00 & 0.00 \\
\hline \multirow{4}{*}{$\begin{array}{l}\text { Igual presión tributaria } \\
\text { (2) }\end{array}$} & Rec. propios per cápita & 0.26 & 0.25 & 0.26 \\
\hline & Transf. Per cápita & 0.18 & 0.07 & 0.10 \\
\hline & Presión tributaria & 0.00 & 0.00 & 0.00 \\
\hline & Gasto per cápita & 0.20 & 0.12 & 0.15 \\
\hline \multirow{6}{*}{$\begin{array}{l}\text { Igual gasto per cápita } \\
\text { (3) }\end{array}$} & Correspondencia fiscal & 0.12 & 0.16 & 0.14 \\
\hline & Rec. propios per cápita & 0.08 & 0.20 & 0.07 \\
\hline & Transf. Per cápita & 0.18 & 0.07 & 0.10 \\
\hline & Presión tributaria & 0.26 & 0.39 & 0.25 \\
\hline & Gasto per cápita & 0.00 & 0.00 & 0.00 \\
\hline & Correspondencia fiscal & 0.08 & 0.20 & 0.07 \\
\hline
\end{tabular}

(1) Se simula un escenario donde el gasto total es igual a los recursos propios totales.

(2) el gasto público es ajustado en función de las diferencias entre la presión tributaria de cada jurisdicción y la presión tributaria promedio.

(3) la presión tributaria es ajustada en función de las diferencias entre el gasto per cápita de cada jurisdicción y el gasto per cápita promedio.

\section{CONCLUSIONES}

Brasil presenta descentralización asimétrica de gastos y recursos con desequilibrio vertical, que es particularmente importante en el nivel municipal. También es importante el grado de descentralización fiscal subnacional (gastos y recursos municipales/gastos y recursos subnacionales). La estructura vertical es muy heterogénea entre los Estados.

La descentralización subnacional, tanto de gastos como de recursos propios, es mayor en los Estados con mayor población: la mayor importancia relativa de las municipalidades en los estados más poblados permite adaptar mejor la oferta a la demanda de bienes municipales a la vez que el costo se reparte en un grupo de mayor tamaño (economías de escala). El coeficiente del PBGpc es positivo y significativo para los gastos y los recursos propios: en los estados con mayor PBGpc los recursos propios están más descentralizados. Estos resultados pueden 
ser importantes para la política fiscal hacia el futuro. Si se prevé que las municipalidades tendrán una participación creciente dentro del sector público subnacional, la modernización y fortalecimiento municipal debería ocupar un lugar importante en la agenda de las políticas públicas.

El gasto per cápita de los Estados, las municipalidades y el agregado subnacional aumentan con el tamaño de la población, siendo mayor el coeficiente correspondiente a las municipalidades. Algo similar ocurre con los recursos propios. Los gastos y recursos propios de los estados, municipalidades y el total subnacional, se relacionan positivamente con el PBGpc, indicando que se trata de bienes "normales" y que los recursos propios aumentan con el PBGpc. Como ocurría con la población, los coeficientes de las municipalidades son mayores que los de los Estados.

Existen grandes disparidades horizontales en cuanto a gastos y recursos propios. Esto implica que no existe igualdad en cuanto a la disponibilidad de bienes públicos subnacionales (medida utilizando el gasto).

La autonomía (correspondencia) fiscal varía entre los GSN intermedios y las municipalidades. Para el año 2012 la relación entre los valores máximo y mínimo de la correspondencia fiscal normalizada fue, a nivel de los GSN intermedios, de 4 veces y para los municipios de 6,6 veces.

Dada la amplia heterogeneidad entre los GSN intermedios y municipales en cada país, es recomendable que en el diseño e implementación de las políticas fiscales subnacionales se adapte a las particularidades de cada unidad territorial evitando generalizar políticas públicas por nivel de gobierno.

En base a la información presupuestaria, comparando las elasticidades-PBG de los gastos y los recursos propios subnacionales, las estimaciones cross section sugieren un rol igualador de las transferencias que no se verifica en el modelo de efectos fijos. Una forma más adecuada de cuantificar el efecto igualador resulta de calcular la diferencia entre las transferencias recibidas y el aporte realizado para su financiamiento. El aporte neto es mayor cuanto mayor el PBGpc de las jurisdicciones de modo que se verifica la presencia del efecto igualador.

Se calcularon los efectos de políticas fiscales alternativas sobre las variables fiscales de los GSN. Una política de autonomía total, ceteris paribus, implicaría un importante aumento de las disparidades de gasto per cápita. La aplicación de una política de igualación de la presión tributaria en todas las jurisdicciones tendría impactos diferenciales en los estados y las municipalidades; en los departamentos aumentarían las diferencias en recursos propios y gastos en tanto que en las municipalidades disminuirían. Por otro lado, si el objetivo de la política fuera igualar el gasto per cápita con transferencias constantes, se incrementaría en forma importante la desigualdad observada en la presión tributaria. Finalmente, si las transferencias fueran el único medio de financiamiento y se distribuyeran en base a la población (igual per cápita) se lograría la igualación, pero con niveles absolutos de gasto per cápita que difieren notablemente de la situación real. Estas simulaciones revelan "trade-offs" entre objetivos ya que lograr la igualdad horizontal en una dimensión lleva a incrementar la 
desigualdad en otras planteando un desafío para el diseño de políticas.

Las cuantificaciones y estimaciones pueden ser de utilidad al momento de diseñar y evaluar políticas para el sector subnacional. Las políticas deben comprender gastos, recursos propios y transferencias, considerando los conflictos entre los objetivos de eficiencia y equidad y el efecto sobre los incentivos, la solvencia y la sostenibilidad fiscal. El avance de la descentralización subnacional debe también enfrentar condicionantes de tipo político (del mismo modo que el gobierno nacional interfiere en los gobiernos subnacionales, éstos interfieren en las municipalidades) como las ilustradas, entre otros, en los trabajos de Gomes (2012), Rezende (2016) y Carvalho y Silveira Neto (2018).

Algunas limitaciones del trabajo deben tenerse en cuenta. Una es que utiliza datos agregados para las variables fiscales (gastos, recursos propios y transferencias) lo que oculta que los componentes pueden tener comportamientos distintos. A modo de ejemplo, no todos los gastos (y lo mismo con los recursos propios y las transferencias) tienen el mismo efecto sobre la equidad regional. Del mismo modo se trabajó con el universo de municipalidades y estados que son un conjunto muy heterogéneo, pudiendo resultar que las variables fiscales se comporten de manera distinta según las características socio-económicas y demográficas de esas jurisdicciones. La metodología de la investigación consistió, en este punto, en controlar las heterogeneidades con las variables población, superficie y PBGpc. Se utilizó también el modelo econométrico de Efectos Fijos para controlar por las heterogeneidades no observables de cada jurisdicción. Queda para la agenda de investigación avanzar en análisis desagregados que brindarán resultados adicionales a los presentados en este trabajo que, pese a las limitaciones, es de utilidad para diseñar y evaluar políticas sobre descentralización.

\section{REFERENCIAS}

Afonso, J. R., \& J. Serra. (2007). El federalismo fiscal en Brasil: Una visión panorámica. Revista CEPAL, (91), 29-52.

Bahl R., \& S. Wallace. (2003). Fiscal decentralization: The provincial-local dimension. In J. Martinez-Vázquez \& J. Alm. (Eds.), Public finance in developing and transitional countries (pp. 5-34). E. Elgart.

Bell, D. (1987). The world and the United States in 2013. Daedalus, 116(3), 1-31.

Bird, R. M. (2001). Setting the stage: Municipal and intergovernmental finance. In M. E. Freire \& R. E. Stern (Eds.), The challenge of urban governance (pp. 113-128). World Bank Institute. USA.

Bird, R. M. (2010). Subnational taxation in developing countries. Policy Research Working Paper 5450, The World Bank.

Bird, R. M., \& Slack, E. (2014). Local taxes and local expenditures in developing countries: Strengthening the Wicksellian connection. Public Administration and Development, 34(5), 359-369. doi:10.1002/pad.1695.

Brennan, G., \& Buchanan J. M. (1977). Towards a tax constitution for Leviathan. Journal of Public Economics, 8(3), 255-273. doi:10.1016/0047-2727(77)90001-9 
Brueckner, J. K. (2004). Fiscal decentralization with distortionary taxation: Tiebout vs. tax competition. International Tax and Public Finance, 11(2), 133-153. doi:10.1023/ B:ITAX.0000011397.47855.7c

Buchanan, J. (1950). Federalism and fiscal equity. American Economic Review, 40(4), 583-599.

Carvalho de Andrada Lima, R., \& da Mota Silveira Neto, R. (2018). Secession of municipalities and economies of scale: Evidence from Brazil. Journal of Regional Science, 58(1), 159-180. doi:10.1111/jors. 12348

Courchene, T. J. (1993). Glocalization, institutional evolution, and the Australian Federation. In B. Calligan (Ed.), Federalism and the economy: International, National and State issues. Federalism Research Center, Australian National University.

Genschel, P., \& Schwartz, P. (2011). Tax competition: A literature review. Socio-Economic Review, 9(2), 339-370. doi:10.1093/ ser/mwr004

Gomes, S. (2012). Fiscal powers to subnational governments: Reassessing the concept of fiscal autonomy. Regional \& Federal Studies, 22(4), 387-406. doi:10.1080/13597566 .2012 .679849

Grin, E. J. \& Abrucio, F. L. (2016). Facetas del federalismo en Brasil: descentralización, recentralización y los desafíos de la cooperación intergubernamental. Revista lberoamericana de Gobierno Local, Artículo de Divulgación, No 11. (11).
Oakland, W. H. (1994). Recognizing and correcting for fiscal disparities. In J. E. Anderson (Ed.), Fiscal equalization for state and local government finance (pp. 1-19). Westport: Praeger.

Oates, W. E. (1972). Fiscal Federalism, Harcourt Brace Jovanovich, Inc. Versión en español del Instituto de Administración Local, Madrid, 1977, con el título Federalismo Fiscal.

Oates, W. E. (1985). Searching for Leviathan: An empirical study. American Economic Review, 75(4), 748-757.

Oates, W. E. (1988). On the measurement of congestion in the provision of local public goods. Journal of Urban Economics, 24(1), 85-94. doi:10.1016/0094-1190(88)90048-4.

Oates, W. E. (2005). Toward a second-generation theory of fiscal federalism. International Tax and Public Finance, 12(4), 349-373. doi:10.1007/s10797-005-1619-9.

Porto, A., Pineda Mannheim, C., \& Eguino, H. (2018). Descentralización y autonomía fiscal en América Latina: Panorama comparado de Brasil, Colombia, México y Perú. Documento para Discusión No IDB-DP-557.

Qian, Y., \& Weingast, B. R. (1997). Federalism as a commitment to preserving market incentives. The Journal of Economic Perspectives, 11(4), 83-92.

Rezende, F. (2016). Conflitos Federativos, Ed. Fórum, Belo Horizonte, Brasil.

Tiebout, C. M. (1956). A pure theory of local expenditures. Journal of Political Economy, 64(5), 416-424. 
Villela, L., Afonso, J. R., \& Calvo, A. (2016). Transferencias intergubernamentales en Brasil. Revista da Academia Brasileira de Direito Constitucional, 8(14), 60-78.

Wallis, J., \& Oates, W. E. (1988). Decentralization in the public sector: An empirical study of State and Local government. In $\mathrm{H}$. Rosen (Ed), Fiscal Federalism. Quantitative Studies (pp. 5-32). Chicago, IL: University of Chicago Press.
Weingast, B. R. (1995). The economic rol of political institutions: Market preserving federalism and economic growth. Journal of Law, Economics and Organization, 11(1), 1-31.

Weingast, B. R. (2009). Second generation fiscal federalism: The implications of fiscal incentives. Journal of Urban Economics, 65(3), 279-293. doi:10.1016/j.jue.2008.12.005. 